\title{
Changes of Tumor Infiltrating Lymphocytes after Core Needle Biopsy and the Prognostic Implications in Early Stage Breast Cancer: A Retrospective Study
}

\author{
Jiahui Huang, $M D^{1}$ \\ Xiaosong Chen, $\mathrm{MD}, \mathrm{PhD}^{1}$ \\ Xiaochun Fei, MD2 \\ Ou Huang, MD, PhD' \\ Jiayi $\mathbf{W u}, M D, P h D^{1}$ \\ Li Zhu, MD, PhD' \\ Jianrong He, MD, PhD ${ }^{1}$ \\ Weiguo Chen, MD \\ Yafen Li, MD' \\ Kunwei Shen, MD, PhD'
}

${ }^{1}$ Comprehensive Breast Health Center, ${ }^{2}$ Department of Pathology, Ruijin Hospital Affiliated to Shanghai Jiao Tong University School of Medicine, Shanghai, China
Correspondence: Kunwei Shen, MD, PhD Comprehensive Breast Health Center, Ruijin Hospital Affiliated to Shanghai Jiao Tong University School of Medicine, 197 Ruijin Er Road, Huangpu District, Shanghai 200025, China

Tel: 86-21-64370045 (602208)

Fax: 86-21-6415-6886

E-mail: kwshen@medmail.com.cn

Received September 11, 2018

Accepted February 7, 2019

Published Online February 12, 2019

\section{Purpose}

The purpose of this study was to investigate the changes of tumor infiltrating lymphocytes (TILs) between core needle biopsy (CNB) and surgery removed sample (SRS) in early stage breast cancer patients and to identify the correlating factors and prognostic significance of TILs changes.

\section{Materials and Methods}

A retrospective study was carried out on 255 patients who received CNB and underwent surgical resection for invasive breast cancer. Stromal TILs levels of CNB and SRS were evaluated respectively. Tumors with $\geq 50 \%$ stromal TILs were defined as lymphocyte-predominant breast cancer (LPBC). Clinicopathological variables were analyzed to determine whether there were factors associated with TILs changes. Log-rank tests and Cox proportional hazards models were used to analyze the influences of TILs and TILs changes on survival.

\section{Results}

SRS-TILs (median, 10.0\%) were significant higher than CNB-TILs (median, 5.0\%; $p<0.001$ ). Younger age ( $<60$ years, $p=0.016)$ and long surgery time interval (STI, $\geq 4$ days; $p=0.003$ ) were independent factors correlating with higher TILs changes. CNB-LPBC patients showed better breast cancer-free interval $(B C F I, p=0.021)$ than CNB-non-LPBC (CNB-nLPBC) patients. Patients were categorized into four groups according to the LPBC change pattern from CNB to SRS: $\mathrm{LPBC} \rightarrow \mathrm{LPBC}, \mathrm{LPBC} \rightarrow \mathrm{nLPBC}, \mathrm{nLPBC} \rightarrow \mathrm{LPBC}$, and $\mathrm{nLPBC} \rightarrow \mathrm{nLPBC}$, with estimated 5-year BCFI 100\%, 100\%, 69.7\%, and 86.0\% ( $p=0.016)$. nLPBC $\rightarrow$ LPBC pattern was an independent prognostic factor of worse BCFI (hazard ratio, 2.19; 95\% confidence interval, 1.06 to 4.53 ; $p=0.035$ ) compared with other patterns.

\section{Conclusion}

TILs were significantly higher in SRS than in CNB. Higher TILs changes were associated with younger age and long STI. Changing from nLPBC to LPBC after CNB indicated a worse BCFI, which needs further validation.

\section{Introduction}

Tumor immune microenvironment plays an important role in tumor development, progression, and control. Effective immune surveillance by the host immune system protects the body against cancer. In breast cancer, lymphocyte

\author{
Key words \\ Breast neoplasms, Core needle biopsy, Surgery, \\ Tumor infiltrating lymphocytes, Prognosis
}

infiltration was detectable in more than $80 \%$ of tumors [1-3], and accumulating evidence from several recent large clinical studies indicates that high levels of tumor infiltrating lymphocytes (TILs) are predictive of better response to neoadjuvant chemotherapy, and increased survival [2-9]. These results suggest that tumors with different TILs levels may have different biologic behaviors and different treatment 
responses, and there is a role to evaluate TILs in clinical trial cohorts as well as in daily histopathological practice. However, as a quantitative histological biomarker, the utilization of TILs depends on the standardization and reproducibility of TILs evaluation. A guideline for evaluation of TILs in breast cancer has been published in 2014 [10], and two multicenter international ring studies have been conducted to make TILs a reproducible marker [11].

Neoadjuvant therapy (NAT) is an increasingly used therapeutic strategy for initially large, inoperable, or locally advanced breast tumors, as well as operable cancers susceptible to specific treatments. NAT not only induce clinical responses that allow breast-conserving surgery and better survival, but also allows observation of tumor response to specific regimen. Sequential biopsies of the same tumor to be taken at different time points during NAT, allows assessment of biologic changes in the tumor long before clinical evidence of response can be determined. The changes of biomarkers like Ki67 after a short exposure to pre-surgery therapy has been found to be related with survival $[12,13]$. Post-treatment infiltration of lymphocytes has also been found to be associated with breast cancer outcome [14-18]. Early detection of changes of TILs during NAT may play a potential role in treatment response evaluation for breast cancer patients.

Core needle biopsy (CNB) is routinely used in breast cancer biopsy. Discordance of biomarkers, including estrogen receptor (ER), progesterone receptor (PR), human epidermal growth factor receptor 2 (HER2), and Ki67 exist between CNB and surgery removed samples (SRS) [19,20]. Tumor heterogeneity, the process of specimen fixation and the procedure of $\mathrm{CNB}$ can cause discordance of biomarkers evaluation between CNB and SRS [21-23]. If we observed changes of biomarkers in sequential biopsies, we need to consider whether the variations have occurred as a result of treatment, but not for other reasons. We need to confirm that the information we got from the core biopsies can represent the whole tumor. However, there is limited data on whether TILs status in CNBs can represent those in the whole tumor section. To solve this question, the present study was designed to compare the TILs status between sequential CNB samples and SRS of breast cancer patients who received surgery soon after CNB without NAT, to find which factors are related with TILs changes, and to further explore the prognostic significance of TILs and TILs changes.

\section{Materials and Methods}

\section{Patients}

We retrospectively analyzed patients who received CNB and underwent the following surgical resection for breast cancer between January 2009 and December 2011 in Comprehensive Breast Health Center, Shanghai Ruijin Hospital. The CNB procedure was done under the guide of ultrasound and at least four 14-gauge CNB samples were collected for pathological examination.

Patient who met all the following criteria were included in this study: (1) female sex; (2) found invasive carcinoma in both CNB and SRS; (3) no preoperative anti-cancer therapy; and (4) paired CNB and SRS sections available for TILs evaluation. Patients with stage IV breast cancer, with bilateral breast cancer or with other malignant diseases at the time of breast cancer diagnosis were excluded from this study.

The baseline clinicopathological characteristics of the patients were achieved from Shanghai Jiaotong University Breast Cancer Database, including age, menopausal status, performing date of CNB and definite excision surgery, surgery type, and histopathological information. Patients received adjuvant treatments according to physicians' choices. Adjuvant treatment information and survival status were obtained through outpatient medical history and/or phone calls.

\section{Pathologic assessment}

The common histopathological information, including breast cancer pathological type, histological grade, tumor size, lymph node status, immunohistochemical (IHC) results of ER, PR, HER2, and Ki67, were achieved from patients' medical records. ER positivity $(\mathrm{ER}+)$ and $\mathrm{PR}$ positivity $(\mathrm{PR}+)$ were defined as $1 \%$ or more positive invasive tumor cells with nuclear staining. Hormonal receptor positivity $(\mathrm{HR}+)$ was defined as ER+ or PR+. HER2 status was firstly determined by IHC staining and scored as $0,1+, 2+, 3+$ according to the 2007 American Society of Clinical Oncology/College of American Pathologists (ASCO/CAP) guideline. Samples with HER2 IHC 2+ were further examined by fluorescence in situ hybridization (FISH). Tumors with HER2 IHC 3+ or FISH positive were regarded as HER2 positive. Five breast cancer molecular subtypes were classified according to the 2013 St. Gallen breast cancer consensus [24]: luminal A (ER+/HER2-, Ki67 $<14 \%$ and PR $\geq 20 \%$ ), luminal B-HER2(ER+ / HER2-, Ki67 $\geq 14 \%$, or ER+/HER2-, PR $<20 \%$, or ER/ PR+ / HER2-), luminal B-HER2+ (HR+/HER2+), triple-negative (TN, HR-/HER2-), and HER2+ (HR-/HER2+).

Evaluations of stromal TILs were performed on readymade full-face hematoxylin and eosin stained sections of 
Table 1. Baseline patient characteristics

\begin{tabular}{|c|c|}
\hline Characteristic & No. $(\%)(\mathrm{n}=255)$ \\
\hline \multicolumn{2}{|l|}{ Age (yr) } \\
\hline$<40$ & $16(6.3)$ \\
\hline $40-49$ & $60(23.5)$ \\
\hline $50-59$ & $78(30.6)$ \\
\hline $60-69$ & $61(23.9)$ \\
\hline$\geq 70$ & 40 (15.7) \\
\hline \multicolumn{2}{|l|}{ Menstrual status } \\
\hline Premenopausal & $86(33.7)$ \\
\hline Postmenopausal & $169(66.3)$ \\
\hline \multicolumn{2}{|l|}{ Breast surgery type } \\
\hline Mastectomy & $223(87.5)$ \\
\hline Breast-conserving surgery & $32(12.5)$ \\
\hline \multicolumn{2}{|l|}{ Pathological type } \\
\hline Invasive ductal carcinoma & $241(94.9)$ \\
\hline Invasive lobular carcinoma & $9(3.5)$ \\
\hline Others & $5(1.6)$ \\
\hline \multicolumn{2}{|l|}{ Histological grade } \\
\hline I-II & $132(51.8)$ \\
\hline III & $110(43.1)$ \\
\hline NA & $13(5.1)$ \\
\hline \multicolumn{2}{|l|}{ Tumor size $(\mathrm{cm})$} \\
\hline$\leq 2$ & $106(41.6)$ \\
\hline $2-5$ & $139(54.5)$ \\
\hline$>5$ & $10(3.9)$ \\
\hline \multicolumn{2}{|l|}{ Axillary lymph node status } \\
\hline Negative & $131(51.4)$ \\
\hline 1-3 Positive & $75(29.4)$ \\
\hline 4-9 Positive & $24(9.4)$ \\
\hline$>10$ Positive & $25(9.8)$ \\
\hline \multicolumn{2}{|l|}{ ER } \\
\hline Negative & $66(25.9)$ \\
\hline Positive & $189(74.1)$ \\
\hline \multicolumn{2}{|l|}{ PR } \\
\hline Negative & $93(36.5)$ \\
\hline Positive & $162(63.5)$ \\
\hline \multicolumn{2}{|l|}{ HER2 } \\
\hline Negative & $204(80.0)$ \\
\hline Positive & $51(20.0)$ \\
\hline \multicolumn{2}{|l|}{ Ki67 (\%) } \\
\hline$<20$ & $86(33.7)$ \\
\hline$\geq 20$ & $169(66.3)$ \\
\hline \multicolumn{2}{|l|}{ Molecular subtype } \\
\hline Luminal A & $36(14.1)$ \\
\hline Luminal B-HER2- & $130(51.0)$ \\
\hline Luminal B-HER2+ & $25(9.8)$ \\
\hline HER2 enriched & $26(10.2)$ \\
\hline Triple negative & $38(14.9)$ \\
\hline \multicolumn{2}{|l|}{ Surgery time interval (day) } \\
\hline$<4$ & $104(40.8)$ \\
\hline$\geq 4$ & $151(59.2)$ \\
\hline
\end{tabular}

(Continued)
Table 1. Continued

$\begin{array}{lr}\text { Characteristic } & \text { No. }(\%)(\mathbf{n}=255) \\ \text { Chemotherapy } & 194(76.1) \\ \text { Yes } & 61(23.9) \\ \quad \text { No } & 103(40.4) \\ \text { Radiotherapy } & 152(59.6) \\ \text { Yes } & \\ \text { No } & 30(11.8) \\ \text { Targeted therapy (trastuzumab) } & 225(88.2) \\ \quad \text { Yes } & \\ \text { No } \\ \text { Endocrine therapy } \\ \quad \text { Yes } \\ \text { No }\end{array}$

Pathological data is based on surgery removed sample. ER, estrogen receptor; PR, progesterone receptor; HER2, human epidermal growth factor receptor 2.

paraffin-embedded CNB and SRS according to the 2014 recommendation of international TILs working group [10] by an experienced pathologist (X.C. Fei). TILs were reported for the stromal compartment ( $=\%$ stromal TILs).

TILs levels were evaluated separately for CNB and SRS, recorded as CNB-TILs and SRS-TILs. Tumors with $\geq 10 \%$ stromal TILs were regarded as high-TILs tumors, while tumors with $<10 \%$ TILs were regarded as low-TILs tumors. Tumors with $\geq 50 \%$ stromal TILs were defined as lymphocyte-predominant breast cancer (LPBC) [2-5,10], while tumors with $<50 \%$ TILs were defined as non-lymphocytepredominant breast cancer (nLPBC). Tumors were categorized into CNB-High-TILs, CNB-Low-TILs, SRS-High-TILs, SRS-Low-TILs, CNB-LPBC, SRS-LPBC, CNB-nLPBC, and SRS-nLPBC according to CNB-TILs and SRS-TILs status.

\section{Statistical analysis}

Change of stromal tumor infiltrating lymphocytes ( $\Delta$ TILs) was defined as the value of SRS-TILs minus CNB-TILs of each patient. Surgery time interval (STI) was defined as the day interval between CNB and definite surgery. Breast cancer-free interval (BCFI) was defined as the interval from the first day after surgery to the date of any of the following events happened: breast cancer recurrence at local or regional sites, metastasis to distant sites or newly diagnosed breast cancer in the contralateral breast. Overall survival (OS) was defined as time from the date of definite surgery to the date of death from any cause.

Kappa test was used to calculate the concordance rate of ER, PR, HER2, Ki67, and TILs between CNB and SRS. Values of $\kappa>0.6$ were correlated with good agreement, values bet- 
ween 0.4 and 0.6 considered moderate agreements, values $<0.4$ corresponded to fair, and values $<0.2$ reflected poor agreement. TIL change after CNB was compared by using the Wilcoxon signed-rank test. Mann-Whitney U test and Kruskal-Wallis test were used to calculate the association between CNB-TILs, SRS-TILs, and $\triangle$ TILs with clinicopathological factors. Multivariate ANOVA analysis was then done to find the association and interaction between the rank of $\Delta$ TILs and the tested factors. Kaplan-Meier method was used to estimate survival curves and log-rank test was used to compare survival between groups. Multivariate Cox proportional hazards models were used to estimate the hazard ratio, 95\% confidence interval $(\mathrm{CI})$, and the effects of clinicopathological variables. All statistical tests were two-sided and $\mathrm{p}<$ 0.05 was considered as statistically significant. All the analyses were done by SPSS ver. 23.0 for Mac (IBM Corp., Armonk, NY).

\section{Ethical statement}

All procedures performed in studies involving human participants were in accordance with the ethical standards of the independent Ethical Committee / Institutional Review Board of Ruijin Hospital and with the 1964 Helsinki declaration and its later amendments or comparable ethical standards. Informed consent was exempted due to the retrospective format of this study.

\section{Results}

\section{Patient characteristics}

The clinicopathological characteristics of the 255 patients included in this study are detailed in Table 1 . The median patients' age was 56 years (range, 28 to 86 years). Mastectomy and breast-conserving surgery were performed in 223 $(87.5 \%)$ and $32(12.5 \%)$ patients, respectively. Tumor size was pT1 $(\leq 2 \mathrm{~cm})$ in $41.6 \%$ and pT2 $(2-5 \mathrm{~cm})$ in $54.5 \%$ patients. The majority (94.1\%) of the patients had an invasive ductal carcinoma and $43.1 \%$ of the patients had a grade III tumor. Axillary lymph node status was pN0 in $51.4 \%$ and pN1 (1-3 positive nodes) in $29.4 \%$ of the cases; the remaining $19.2 \%$ of the patients had four or more metastatic lymph nodes. Most (74.1\%) of the patients had an ER-positive tumor and 20\% had a HER2-positive tumor. Median Ki67 was 20\%. There were $14.1 \%, 51.0 \%, 9.8 \%, 10.2 \%$, and $14.9 \%$ patients classified as luminal A, luminal B-HER2-, luminal B-HER2+, HER2+, and TN subtype, respectively. Median STI was 4 days, ranging from 1 to 32 days. Forty point eight percent of patients received surgery within 1-3 days after CNB. Seventy-six point one percent of the patients received chemotherapy; $40.4 \%$ received radiotherapy; $74.5 \%$ received endocrine therapy; $11.8 \%$ received trastuzumab as targeted therapy, accounting for $58.8 \%(30 / 51)$ of all HER2-positive patients.

\section{Association of TILs with clinicopathological characteris- tics}

The distribution of CNB-TILs and SRS-TILs by different subtypes is shown in Fig. 1A. Overall, the median value of CNB-TILs was $5.0 \%$ (interquartile range [IQR], $1.0 \%$ to $20.0 \%$ ), and $10.2 \%$ of the patients had CNB-LPBC (TILs $\geq 50 \%$ in CNB samples). CNB-TILs were correlated with molecular subtypes $(p<0.001)$, high histological grade $(p<0.001)$, ER negativity $(p<0.001)$, and high Ki67 expression $(\geq 20 \%$, $\mathrm{p}=0.003$ ). The median values of CNB-TILs of luminal A, luminal B-HER2-, luminal B-HER2+, HER2+, and TN were $1.0 \%$ (IQR, $0.0 \%$ to $5.0 \%$ ), $5.0 \%$ (IQR, $1.0 \%$ to $16.3 \%$ ), $8.0 \%$ (IQR, $1.0 \%$ to $25.0 \%$ ), $8.0 \%$ (IQR, $2.8 \%$ to $16.3 \%$ ), and $20.0 \%$ (IQR 5.0 to 50.0), respectively ( $\mathrm{p}<0.001)$. CNB-LPBC phenotype comprised $2.8 \%, 6.9 \%, 12.0 \%, 11.5 \%$, and $26.3 \%$ in luminal A, luminal B-HER2-, luminal B-HER2+, HER2+, and TN subtypes, respectively $(\mathrm{p}=0.004)$. CNB-TILs were not statistically associated with age, menopausal status, lymph node involvement, tumor size, PR status or HER2 status $(p>0.05)$.

The median value of SRS-TILs was $10.0 \%$ (IQR, $5.0 \%$ to $35.0 \%$ ), and $22.0 \%$ of the patients had SRS-LPBC (TILs $\geq 50 \%$ in SRS). SRS-TILs were associated with molecular subtype $(p<0.001)$, high histological grade $(p<0.001)$, ER negativity $(\mathrm{p}<0.001)$, PR negativity $(\mathrm{p}=0.036)$, high Ki67 expression ( $\geq 20 \%, p<0.001)$, younger age $(<60$ years, $p=0.014)$, and long STI ( $~ \geq 4$ days, $p=0.006$ ). The median values of SRS-TILs of luminal A, luminal B-HER2-, luminal B-HER2+, HER2+, and $\mathrm{TN}$ were $5.0 \%$ (IQR, 3.0\% to $15.0 \%$ ), $10.0 \%$ (IQR, $5.0 \%$ to $30.0 \%$ ), $10.0 \%$ (IQR, $4.0 \%$ to $55.0 \%$ ), $20.0 \%$ (IQR, $7.3 \%$ to $56.3 \%$ ), and $35.0 \%$ (IQR, 18.8 to 60.0 ), respectively ( $<<0.001$ ). SRS-LPBC phenotype comprised $5.6 \%, 14.6 \%, 28.0 \%, 38.5 \%$, and $47.4 \%$ in the luminal A, luminal B-HER2-, luminal B-HER2+, HER2+, and TN subtypes, respectively $(\mathrm{p}<0.001)$. SRS-TILs were not statistically associated with menopausal status, lymph node involvement, tumor size, or HER2 status $(\mathrm{p}>0.05)$.

\section{Comparison of ER, PR, HER2, Ki67, and TILs between CNB and SRS}

The concordance rates of ER, PR, and HER2 between CNB and SRS were $94.1 \%$ ( $\kappa=0.841), 87.1 \%(\kappa=0.724)$, and $97.3 \%$ $(\kappa=0.911)$, respectively. CNB and SRS had a good concordance in ER, PR, and HER2.

Ki67 value was much higher in SRS comparing with CNB 


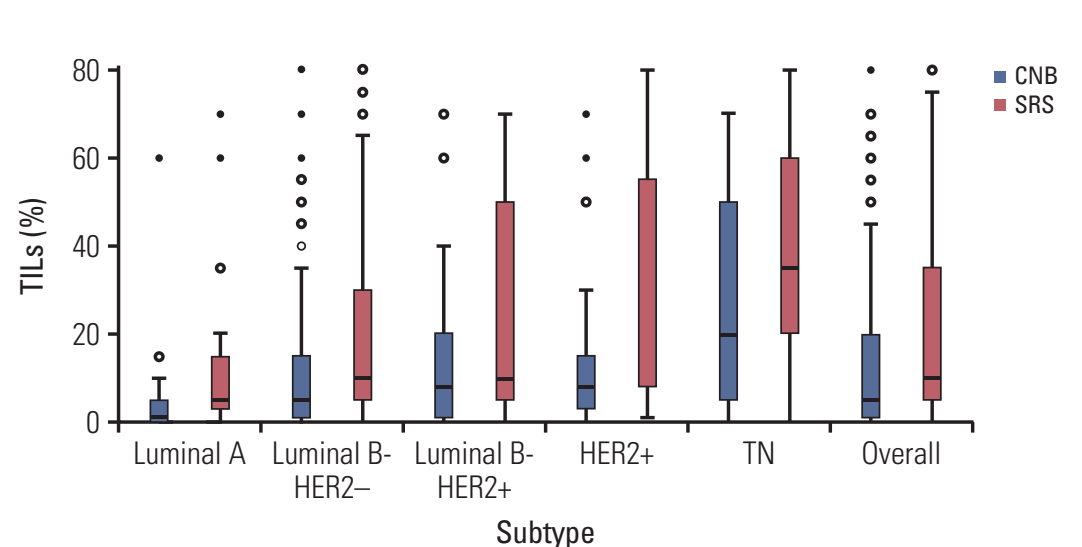

A

\begin{tabular}{rrrrrrrcccccc}
$\mathrm{n}$ & \multicolumn{3}{c}{36} & \multicolumn{1}{c}{130} & \multicolumn{2}{c}{25} & \multicolumn{2}{c}{26} & \multicolumn{2}{c}{28} & \multicolumn{2}{c}{255} \\
Min & 0 & 0 & 0 & 0 & 0 & 0 & 0 & 1 & 0 & 0 & 0 & 0 \\
01 & 0 & 3 & 1 & 5 & 1 & 4 & 2.8 & 7.3 & 5 & 18.8 & 1 & 5 \\
02 & 1 & 5 & 5 & 10 & 8 & 10 & 8 & 20 & 20 & 35 & 5 & 10 \\
03 & 5 & 15 & 16.3 & 30 & 25 & 55 & 16.3 & 56.3 & 50 & 60 & 20 & 35 \\
Max & 60 & 70 & 80 & 80 & 70 & 70 & 70 & 80 & 70 & 80 & 80 & 80
\end{tabular}

B
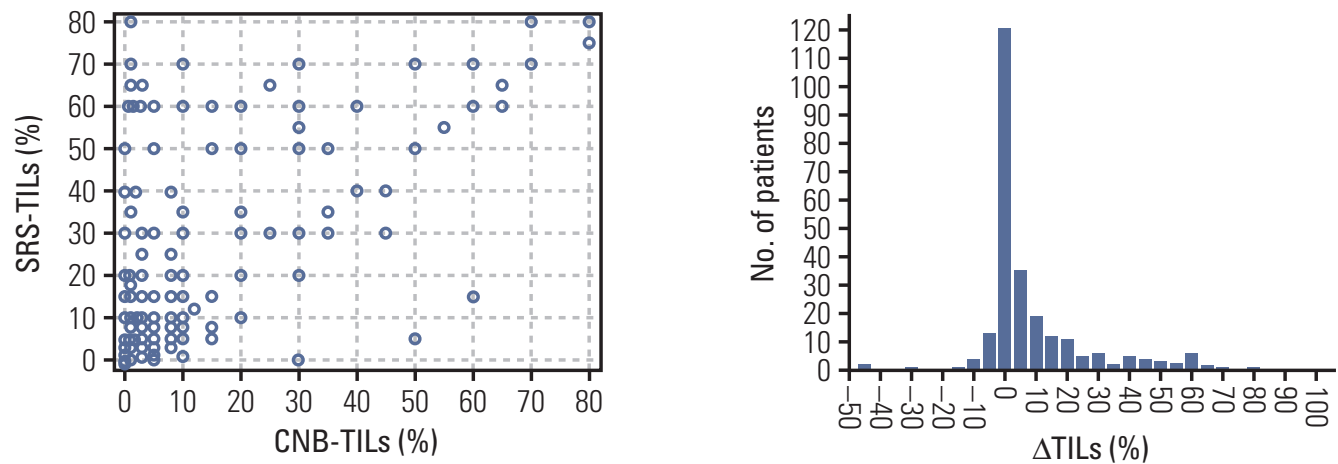

Fig. 1. Distribution of TILs and $\triangle$ TILs. (A) Distribution of CNB-TILs\% and SRS-TILs\% in the global population and per breast cancer subtype. The box shows the limits of the middle half of the data (1st to 3rd quartile), and the line inside represents the median. Whiskers are drawn to represent values within 1.5 times the length of the box from either end of the box (1.5 times the interquartile range). Open circles correspond to mild outliers. Asterisks correspond to extreme outliers. (B) Distribution of CNB-TILs and SRS-TILs per patient. Each open circle represents one patient; (C) Distribution of $\Delta$ TILs (per $10 \%$ ). TILs, tumor infiltrating lymphocytes; $\Delta$ TILs, change of TILs; CNB, core needle biopsy; SRS, surgery removed sample, Max, maximum; Min, minimum; Q, quartile.

samples by using two-paired samples $t$ test, with mean values of $29.5 \%$ and $26.4 \%$, respectively $(p<0.001)$. Take $\geq 20 \%$ as the cutoff value of high Ki67, the concordance rate of Ki67 was $82.4 \%$ ( $\mathrm{k}=0.635)$.

The distribution of CNB-TIL and SRS-TIL of each patient was shown in Fig. 1B. The median and mean value of CNBTILs was $5.0 \%$ and $13.8 \%$, while in SRS-TILs was $10.0 \%$ and $22.3 \%$. SRS-TILs were significantly higher than CNB-TILs by using the Wilcoxon test $(\mathrm{p}<0.001)$. Respectively use $\geq 10 \%$, $\geq 20 \%, \geq 30 \%, \geq 40 \%, \geq 50 \%, \geq 60 \%$, and $\geq 70 \%$ as the cutoff value of high TILs and low TILs, the concordance rates between CNB-TILs and SRS-TILs were 75.3\% ( $\kappa=0.522), 82.4 \%$ $(\kappa=0.607), 82.4 \%$ ( $\kappa=0.554), 85.5 \%$ ( $\kappa=0.525), 86.3 \%$ ( $\kappa=0.500)$, $91.0 \%(\kappa=0.579)$, and $98.4 \%(\kappa=0.593)$. The agreement of TILs between CNB and SRS was just moderate.

$\Delta$ TILs ranged from $-45.0 \%$ to $+79.0 \%$ (Fig. 1C), with median value of $1.0 \%$, and mean value of $8.6 \%$. High $\Delta$ TILs was correlated with younger age ( $<60$ years, $\mathrm{p}=0.016)$, ER negativity $(\mathrm{p}=0.006)$, and long STI ( $\geq 4$ days, $\mathrm{p}=0.003$ ) (Table 2$)$. Multivariate ANOVA analysis showed that younger age $(<60$ 
Table 2. $\Delta$ TILs among patients with different clinicopathological characteristics

\begin{tabular}{|c|c|c|c|}
\hline \multirow{2}{*}{ Characteristic } & \multicolumn{2}{|c|}{$\Delta$ TILs $(\%)$} & \multirow{2}{*}{ p-value } \\
\hline & Median (IQR) & Mean \pm SD & \\
\hline \multicolumn{4}{|l|}{ Age (yr) } \\
\hline$<60$ & $2.0(0.0-17.0)$ & $11.1 \pm 19.6$ & 0.016 \\
\hline$\geq 60$ & $0.0(0.0-5.0)$ & $4.9 \pm 13.3$ & \\
\hline \multicolumn{4}{|l|}{ Menstrual status } \\
\hline Premenopausal & $0.0(0.0-15.0)$ & $11.2 \pm 20.5$ & 0.888 \\
\hline Postmenopausal & $1.0(0.0-10.0)$ & $7.24 \pm 15.8$ & \\
\hline \multicolumn{4}{|l|}{ Tumor size (cm) } \\
\hline$\leq 2$ & $0.0(0.0-10.0)$ & $8.0 \pm 17.8$ & 0.517 \\
\hline$>2$ & $1.0(0.0-12.0)$ & $9.0 \pm 17.5$ & \\
\hline \multicolumn{4}{|c|}{ Axillary lymph node status } \\
\hline Negative & $0.0(0.0-10.0)$ & $8.7 \pm 17.3$ & 0.973 \\
\hline Positive & $1.0(0.0-12.0)$ & $8.4 \pm 18.0$ & \\
\hline \multicolumn{4}{|l|}{ Histological grade } \\
\hline I-II & $0.0(0.0-10.0)$ & $7.2 \pm 15.3$ & 0.360 \\
\hline III & $2.0(0.0-20.0)$ & $10.4 \pm 20.5$ & \\
\hline NA & $2.0(0.0-12.5)$ & $7.2 \pm 11.7$ & \\
\hline \multicolumn{4}{|l|}{ ER } \\
\hline Negative & $6.0(0.0-30.0)$ & $15.3 \pm 24.5$ & 0.006 \\
\hline Positive & $0.0(0.0-7.0)$ & $6.2 \pm 13.7$ & \\
\hline \multicolumn{4}{|l|}{ PR } \\
\hline Negative & $2.0(0.0-17.0)$ & $10.5 \pm 20.0$ & 0.110 \\
\hline Positive & $0.0(0.0-10.0)$ & $7.4 \pm 16.0$ & \\
\hline \multicolumn{4}{|l|}{ HER2 } \\
\hline Negative & $1.0(0.0-10.0)$ & $7.5 \pm 15.2$ & 0.602 \\
\hline Positive & $0.0(0.0-27.5)$ & $13.0 \pm 24.7$ & \\
\hline \multicolumn{4}{|l|}{ Ki67 (\%) } \\
\hline$<20$ & $0.0(0.0-5.0)$ & $4.8 \pm 11.5$ & 0.062 \\
\hline$\geq 20$ & $2.0(0.0-15.0)$ & $10.5 \pm 19.8$ & \\
\hline \multicolumn{4}{|l|}{ Molecular subtype } \\
\hline Luminal A & $2.0(0.0-13.0)$ & $6.8 \pm 11.7$ & 0.074 \\
\hline Luminal B-HER2- & $0.0(0.0-7.0)$ & $6.1 \pm 13.4$ & \\
\hline Luminal B-HER2+ & $0.0(0.0-14.5)$ & $8.3 \pm 21.2$ & \\
\hline HER2 positive & $8.5(0.0-38.5)$ & $17.5 \pm 27.4$ & \\
\hline Triple negative & $5.0(0.0-20.5)$ & $13.0 \pm 21.7$ & \\
\hline \multicolumn{4}{|c|}{ Surgery time interval (day) } \\
\hline$<4$ & $0.0(0.0-5.0)$ & $4.9 \pm 15.4$ & 0.003 \\
\hline$\geq 4$ & $2.0(0.0-16.0)$ & $11.1 \pm 18.6$ & \\
\hline
\end{tabular}

Pathological data is based on surgery removed sample. p-value calculated by Mann-Whitney U test. TILs, tumor infiltrating lymphocytes; IQR, interquartile range; SD, standard deviation; ER, estrogen receptor; PR, progesterone receptor; HER2, human epidermal growth factor receptor 2.

years, $\mathrm{p}=0.007$ ), and long STI ( $\geq 4$ days, $\mathrm{p}=0.004$ ) were still significantly associated with $\Delta$ TILs.

\section{Association between TILs and prognosis}

After a median follow-up of 68 months, there were 44 BCFI events being recorded. Twenty-two deaths were recorded in our study until the last follow-up. Fifteen patients died of breast cancer and the other seven died from other reasons.

At univariate survival analysis, CNB-LPBC was a significant predictor of better BCFI compared with CNB-nLPBC patients (estimated 5-year BCFI rate, $100 \%$ vs. $83.6 \%$; $\mathrm{p}=0.021$ ), 
Table 3. CNB-TILs, SRS-TILs and $\triangle$ TILs according to LPBC change pattern

\begin{tabular}{|c|c|c|c|c|c|c|}
\hline \multirow{2}{*}{ LPBC change pattern } & \multicolumn{2}{|c|}{ CNB-TILs $\%$} & \multicolumn{2}{|c|}{ SRS-TILs $\%$} & \multicolumn{2}{|c|}{$\Delta \operatorname{TILs}(\%)$} \\
\hline & Median (IQR) & Mean \pm SD & Median (IQR) & Mean \pm SD & Median (IQR) & Mean \pm SD \\
\hline $\mathrm{nLPBC} \rightarrow \mathrm{nLPBC}(\mathrm{n}=197)$ & $5.0(1.0$ to 10.0$)$ & $7.5 \pm 9.5$ & 8.0 (4.0 to 15.0$)$ & $11.3 \pm 10.7$ & $0.0(0.0$ to 5.0$)$ & $3.8 \pm 8.7$ \\
\hline $\mathrm{nLPBC} \rightarrow \mathrm{LPBC}(\mathrm{n}=33)$ & 10.0 (2.5 to 27.5$)$ & $1.4 \pm 12.9$ & $60.0(50.0$ to 65.0$)$ & $59.2 \pm 7.9$ & $45.0(30.0$ to 59.0$)$ & $44.7 \pm 16.5$ \\
\hline $\mathrm{LPBC} \rightarrow \mathrm{LPBC}(\mathrm{n}=23)$ & $60.0(60.0$ to 70.0$)$ & $63.3 \pm 8.3$ & $65.0(60.0$ to 70.0$)$ & $65.4 \pm 8.8$ & $0.0(0.0$ to 0.0$)$ & $2.2 \pm 5.8$ \\
\hline $\mathrm{LPBC} \rightarrow \mathrm{nLPBC}(\mathrm{n}=2)$ & $55.0(55.0$ to 55.0$)$ & $55.0 \pm 7.1$ & 10.0 (5.0 to 10.0$)$ & $10.0 \pm 7.1$ & $-45.0(-45.0$ to -45.0$)$ & $-45.0 \pm 0.0$ \\
\hline Total & $5.0(1.0$ to 20.0$)$ & $13.8 \pm 19.1$ & 10.0 (5.0 to 35.0$)$ & $22.3 \pm 23.3$ & $1.0(0.0$ to 10.0$)$ & $8.6 \pm 17.6$ \\
\hline
\end{tabular}

CNB, core needle biopsy; TILs, tumor infiltrating lymphocytes; SRS, surgery removed sample; LPBC, lymphocyte-predominant breast cancer; IQR, interquartile range; SD, standard deviation; $\mathrm{nLPBC}$, non-lymphocyte-predominant breast cancer.

while no significant BCFI difference was observed by CNBHigh TILs or CNB-Low TILs ( $\mathrm{p}=0.215$ ), SRS-High TILs or SRS-Low TILs ( $\mathrm{p}=0.457)$, SRS-LPBC or SRS-nLPBC ( $\mathrm{p}=0.752)$.

Patients were categorized into four groups according to the LPBC change pattern from CNB to SRS: LPBC $\rightarrow$ LPBC $(\mathrm{n}=23), \mathrm{LPBC} \rightarrow \mathrm{nLPBC}(\mathrm{n}=2), \mathrm{nLPBC} \rightarrow \mathrm{LPBC}(\mathrm{n}=33)$, and $\mathrm{nLPBC} \rightarrow \mathrm{nLPBC}(\mathrm{n}=197)$. Details of TILs value of CNB and SRS according to LPBC change pattern was listed in Table 3. The estimated 5-year BCFI of patients with $\mathrm{LPBC} \rightarrow \mathrm{LPBC}$, $\mathrm{LPBC} \rightarrow \mathrm{nLPBC}, \mathrm{nLPBC} \rightarrow \mathrm{LPBC}$, and $\mathrm{nLPBC} \rightarrow \mathrm{nLPBC}$ pattern was $100 \%, 100 \%, 69.7 \%$, and $86.0 \%$, respectively $(\mathrm{p}=0.016)$ (Fig. 2A). nLPBC $\rightarrow$ LPBC patients had a worse BCFI than others, $\mathrm{p}=0.013$ (Fig. 2B). We also group patients according to $\Delta$ TILs values $(\Delta$ TILs $<0 \%$ vs. $\geq 0 \%,<5 \%$ vs. $\geq 5 \%,<10 \%$ vs. $\geq 10 \%,<20 \%$ vs. $\geq 20 \%$, and $<30 \%$ vs. $\geq 30 \%$ ) and found no statistical difference of BCFI between groups ( $\mathrm{p}>0.05$, respectively).

In subgroup analysis by molecular subtypes, $\mathrm{nLPBC} \rightarrow$ LPBC pattern was associated with worse BCFI in HER2enriched subtype ( $\mathrm{p}=0.038$ ) but not in luminal $\mathrm{A}$, luminal B-HER2-, luminal B-HER2+, or TN subtypes (Fig. 2C-G). The difference of BCFI according to CNB-LPBC status, SRS-LPBC status, CNB-TILs status (high vs. low) and SRS-TILs (high vs. low) status was not seen in any of the five molecular subtypes.

We generate a multivariate Cox proportional hazards model including tumor size, age, nodal status, histological grade, STI, molecular subtype, CNB-LPBC status, and LPBC change pattern (Table 4) and found LPBC change pattern of $\mathrm{nLPBC} \rightarrow \mathrm{LPBC}$ (hazard ratio, 2.19; 95\% CI, 1.06 to 4.53; $\mathrm{p}=0.035$ ) and tumor size $>2 \mathrm{~cm}$ (hazard ratio, 2.85; 95\% CI, 1.36 to $6.00 ; \mathrm{p}=0.006$ ) were independent prognostic factor of worse BCFI.

There were limited OS events in our study. No significant OS difference was detected between groups by different clinicopathological variables including TILs.

\section{Discussion}

We have already known that variations of biomarkers exist between CNB and SRS, which is supported by multiple studies that investigated the concordance of ER, PR, HER2, and Ki67 status in CNB compared to SRS $[19,20]$. Our results showed a similar concordance of ER, PR, HER2, and Ki67 with our previous study, in which CNB was accurate in evaluating ER, PR, and HER2 while Ki67 value significantly increased after CNB [20]. Few studies pay attention to the variability of immune profiles between CNB and SRS. Jeselsohn et al. [25] have reported activation of immune-related genes after $\mathrm{CNB}$ in a retrospective study evaluating 21 paired core biopsies and excisions from breast cancer patients who did not receive NAT. However, animal experiments conducted by Mathenge et al. [26] showed that tumors from biopsied mice contained a lower frequency of $\mathrm{CD} 4^{+} \mathrm{T}$ cells, $\mathrm{CD}^{+} \mathrm{T}$ cells, and macrophages than that of un-biopsied mice, suggesting biopsy-mediated development of an increasingly immunosuppressive tumor microenvironment [26]. In this study, we found that TILs were higher in SRS than in CNB. The reasons why TILs changes after CNB were hypothetical: (1) with limited evidence, CNB was considered to associate with activation of immune response in breast cancer [25], which indicates the difference between CNB and SRS may be caused by the process of CNB. (2) Core biopsy sample is supposed to contain more tissue from the periphery of the tumor and fewer tissue from the center of the tumor, although knowledge of which area of the tumor the needle targets, is difficult to elucidate and might be regarded as random. Malignant tumors usually develop hypoxic and necrotic cores with higher microvascular density [27], which may result in more immune infiltration in tumor center than in the periphery area. This hypothesis indicates that it might be intra-tumor heterogeneity that caused the difference of TILs between CNB and SRS samples.

We found younger age and long STI were correlated with 
A

B
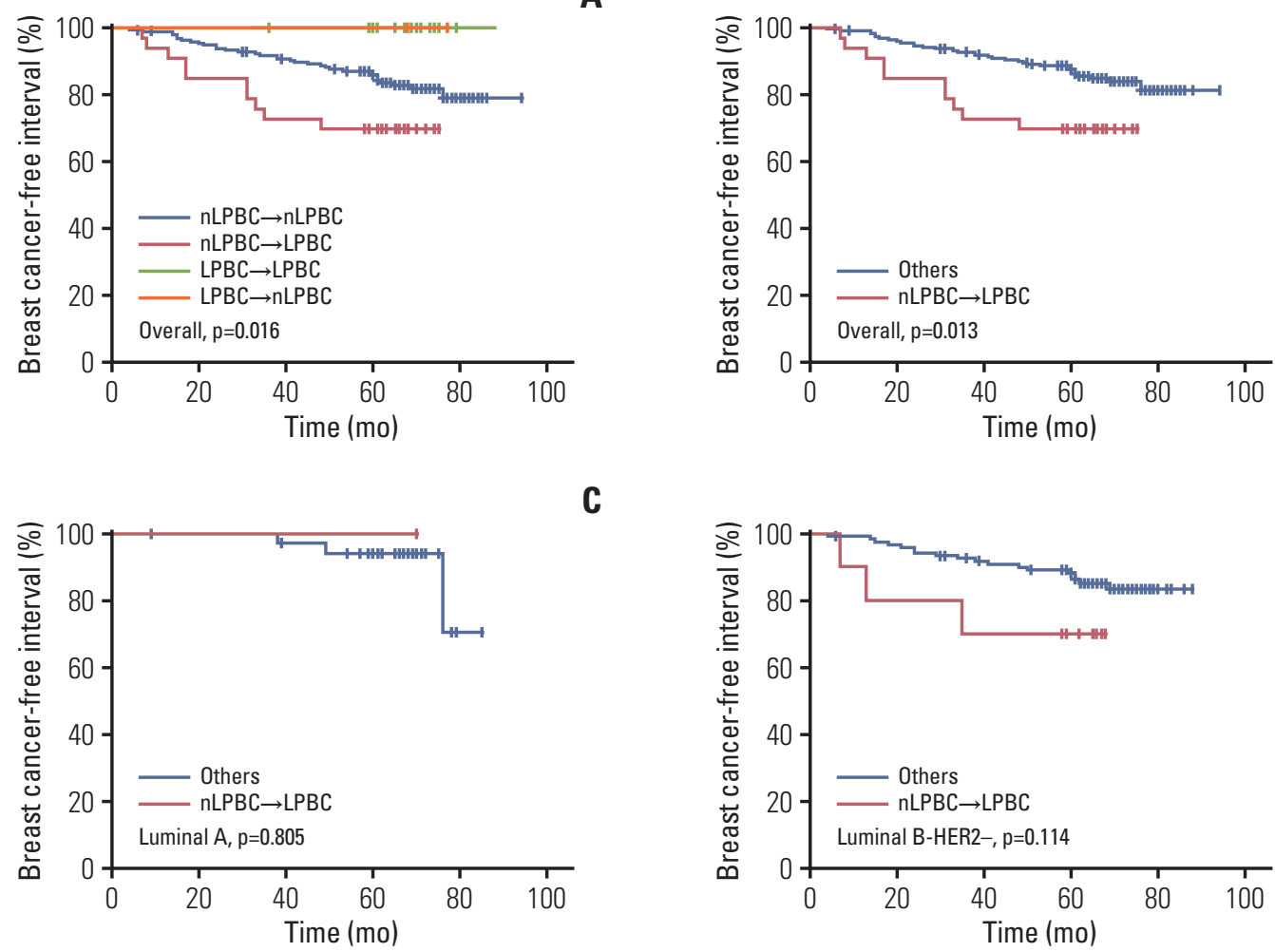

C

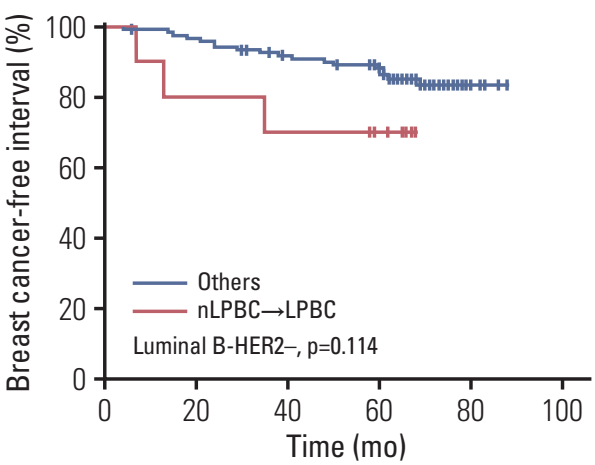

$\mathbf{E}$
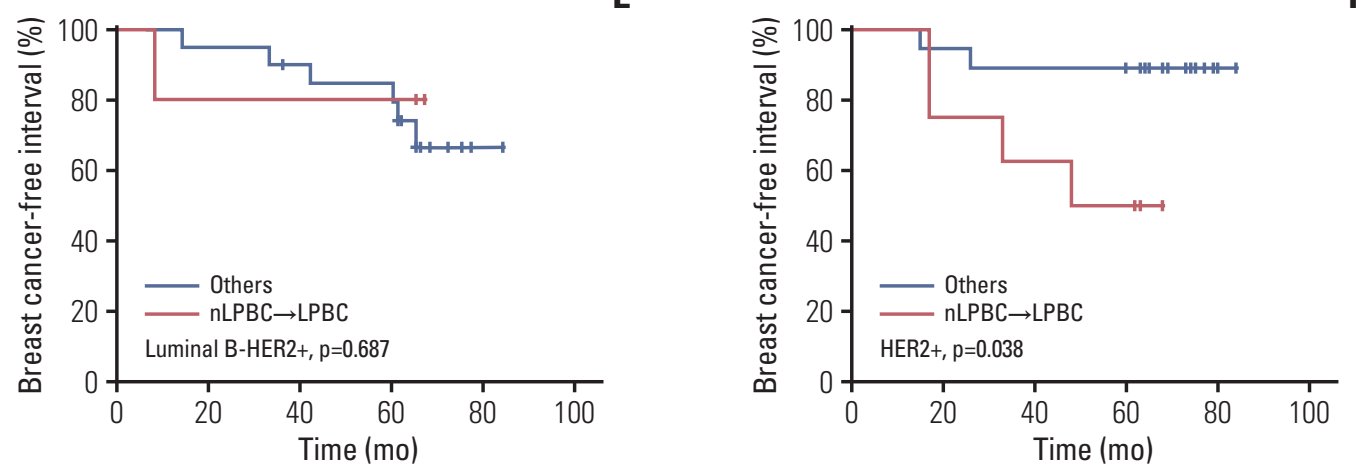

G

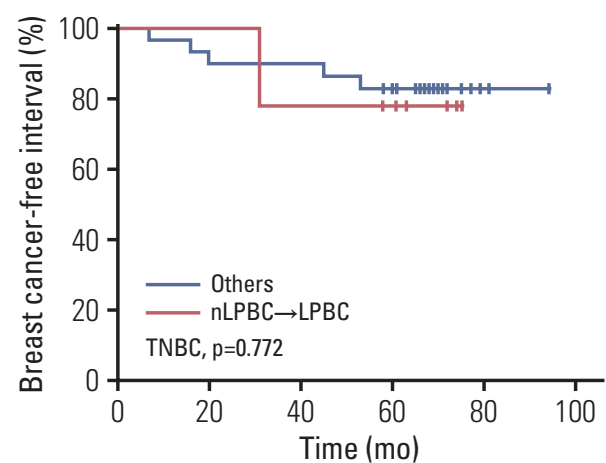

Fig. 2. Breast cancer-free interval by LPBC change status in overall population (A, B), luminal A (C), luminal B-HER2- (D), luminal B-HER2+ (E), HER2+ (F), and TNBC (G) patients. LPBC, lymphocyte predominant breast cancer; nLPBC, non-lymphocyte-predominant breast cancer; HER2, human epidermal growth factor receptor 2; TNBC, triple-negative breast cancer. 
Table 4. Multivariate survival analysis of BCFI

\begin{tabular}{|c|c|c|}
\hline \multirow{2}{*}{ Characteristic } & \multicolumn{2}{|c|}{ BCFI } \\
\hline & p-value & Hazard ratio $(95 \% \mathrm{CI})$ \\
\hline \multicolumn{3}{|l|}{ Age (yr) } \\
\hline$<60$ & 0.156 & 1 \\
\hline$\geq 60$ & & $1.58(0.84-2.96)$ \\
\hline \multicolumn{3}{|l|}{ Tumor size $(\mathrm{cm})$} \\
\hline$<2$ & 0.006 & 1 \\
\hline$\geq 2$ & & $2.85(1.36-6.00)$ \\
\hline \multicolumn{3}{|l|}{ Axillary lymph node status } \\
\hline Negative & 0.230 & 1 \\
\hline Positive & & $1.45(0.79-2.66)$ \\
\hline \multicolumn{3}{|l|}{ Histological grade } \\
\hline I-II & 0.072 & 1 \\
\hline III & & $1.83(0.95-3.51)$ \\
\hline NA & & $3.01(0.99-9.09)$ \\
\hline \multicolumn{3}{|l|}{ Molecular subtype } \\
\hline Luminal A & 0.857 & \\
\hline Luminal B-HER2- & & $1.59(0.46-5.49)$ \\
\hline Luminal B-HER2+ & & $2.21(0.55-8.91)$ \\
\hline HER2 positive & & $1.83(0.42-7.92)$ \\
\hline Triple-negative & & $0.62(0.33-1.17)$ \\
\hline \multicolumn{3}{|l|}{ Surgery time interval (day) } \\
\hline$<4$ & 0.211 & 1 \\
\hline$\geq 4$ & & $0.67(0.36-1.25)$ \\
\hline \multicolumn{3}{|l|}{ CNB-LPBC status } \\
\hline nLPBC & 0.969 & 1 \\
\hline LPBC & & $0.00\left(0.00-8.85^{279}\right)$ \\
\hline \multicolumn{3}{|l|}{ LPBC change pattern } \\
\hline $\mathrm{nLPBC} \rightarrow \mathrm{nLPBC}, \mathrm{LPBC} \rightarrow \mathrm{LPBC}$, or $\mathrm{LPBC} \rightarrow \mathrm{nLPBC}$ & 0.035 & 1 \\
\hline $\mathrm{nLPBC} \rightarrow \mathrm{LPBC}$ & & $2.19(1.06-4.53)$ \\
\hline
\end{tabular}

Pathological data is based on surgery removed sample if not specified. BCFI, breast cancer-free interval; CI, confidence interval; NA, not available; HER2, human epidermal growth factor receptor 2; CNB, core needle biopsy; LPBC, lymphocytepredominant breast cancer; nLPBC, non-lymphocyte-predominant breast cancer.

higher TILs changes after CNB. The level of TILs was more stable in elder patients. Aging is associated with the decrease of autoimmune function, due to the degeneration of thymus gland, the decreased export and replication of T cells, and the reduced immune cell function [28]. For the above reasons, elder patients may have insufficient immune activation after CNB compared with younger patients so that TILs may remain stable. We also assumed that the tumor biological behavior is always milder in elderly patients and tend to generate a more homogeneous tumor, which result in a similar TILs between CNB and SRS. Longer STI was also an indicator of higher chance of TILs increase after CNB in our study. It indicated that the change of TILs after CNB can be dynamic and contribute to the hypothesis that $\mathrm{CNB}$ process caused TILs changes. Further studies are required to verify the difference we found between CNB and SRS and to elucidate the underlying mechanism.

The prognostic value of TILs had been studied in a number of trials. Meta-analysis has indicated that TILs were not prognostic markers for disease-free survival (DFS) and OS in overall population, but related to improved DFS and OS in triple-negative breast cancer (TNBC) [29]. In our study, we failed to detect the association between CNB-TILs or SRSTILs and survival, neither in the overall population nor in the TNBC subgroup. At this point, we cannot conclude whether CNB-TILs and SRS-TILs have equal value in survival prediction and further validation is needed. This is also the first study assessing the correlation between changes of TILs after CNB and survival. We found that patients changed from $\mathrm{nLPBC}$ to $\mathrm{LPBC}$ after CNB experienced a worse BCFI 
than patients with other LPBC change patterns, especially in HER2+ patients. This result was quite different from the common view that TILs increase is related with better survival $[14,16,18]$, although the related studies were mainly conducted in TNBC. In HER2+ tumor, a recent study showed spatial heterogeneity of lymphocytic infiltrate was associated with poor prognosis [30], which is similar with our study. The difference of LPBC status between CNB and SRS may reflect strong regional segregation of immune cells and its association with poor prognosis may indicate dysfunctional immune infiltrate. These results indicated that when we do research about the clinical significance of TILs change during NAT, we need to take account of the difference of TILs between CNB sample and the whole tumor at the time of biopsy.

There were several limitations of this study. The sample size was too small especially when we analyze patients in different subgroups. In addition, it was a retrospective study, the treatments of patients were not standardized, which may affect the result of survival analysis. At last, we only analyzed total TILs without analyzing TILs subsets and were lack of mechanism study. Our next step is to enlarge the sample size and initiate a prospective cohort to validate our results, to figure out the detailed composition of TILs subsets and the changes of gene profiles to study the underlying mechanism.

In summary, our study demonstrated that for patients who received CNB before surgery, TILs were significantly higher in SRS than in CNB. $\triangle$ TILs were associated with younger age and long STI. Patients who change from nLPBC to LPBC after $\mathrm{CNB}$ tend to have the worst $\mathrm{BCFI}$ among the study population, which warrants further validation and further mechanism studies.

\section{Conflicts of Interest}

Conflict of interest relevant to this article was not reported.

\section{Acknowledgments}

This research was supported by the Grants from National Natural Science Foundation of China (grant number: 81472462, 81772797); Medical Guidance Foundation of Shanghai Municipal Science and Technology Commission (grant number: 15411966400); Joint Research Project of the Emerging Cutting-edge Technology of Shanghai Shen-kang Hospital Development Center (grant number: SHDC12014103); Technology Innovation Act Plan of Shanghai Municipal Science and Technology Commission (grant number: $15411952500,15411952501)$. The funding source had no role in the design of this study and will not have any role during its execution, analyses, interpretation of the data, writing assistance, or decision to submit results. The authors thank the patients and the investigators who participated in this study.

\section{References}

1. Lee HJ, Seo JY, Ahn JH, Ahn SH, Gong G. Tumor-associated lymphocytes predict response to neoadjuvant chemotherapy in breast cancer patients. J Breast Cancer. 2013;16:32-9.

2. Loi S, Michiels S, Salgado R, Sirtaine N, Jose V, Fumagalli D, et al. Tumor infiltrating lymphocytes are prognostic in triple negative breast cancer and predictive for trastuzumab benefit in early breast cancer: results from the FinHER trial. Ann Oncol. 2014;25:1544-50.

3. Adams S, Gray RJ, Demaria S, Goldstein L, Perez EA, Shulman $\mathrm{LN}$, et al. Prognostic value of tumor-infiltrating lymphocytes in triple-negative breast cancers from two phase III randomized adjuvant breast cancer trials: ECOG 2197 and ECOG 1199. J Clin Oncol. 2014;32:2959-66.

4. Loi S, Sirtaine N, Piette F, Salgado R, Viale G, Van Eenoo F, et al. Prognostic and predictive value of tumor-infiltrating lymphocytes in a phase III randomized adjuvant breast cancer trial in node-positive breast cancer comparing the addition of docetaxel to doxorubicin with doxorubicin-based chemotherapy: BIG 02-98. J Clin Oncol. 2013;31:860-7.

5. Pruneri G, Vingiani A, Bagnardi V, Rotmensz N, De Rose A,
Palazzo A, et al. Clinical validity of tumor-infiltrating lymphocytes analysis in patients with triple-negative breast cancer. Ann Oncol. 2016;27:249-56.

6. Denkert C, Loibl S, Noske A, Roller M, Muller BM, Komor M, et al. Tumor-associated lymphocytes as an independent predictor of response to neoadjuvant chemotherapy in breast cancer. J Clin Oncol. 2010;28:105-13.

7. Denkert C, von Minckwitz G, Brase JC, Sinn BV, Gade S, Kronenwett $R$, et al. Tumor-infiltrating lymphocytes and response to neoadjuvant chemotherapy with or without carboplatin in human epidermal growth factor receptor 2-positive and triplenegative primary breast cancers. J Clin Oncol. 2015;33:983-91.

8. Salgado R, Denkert C, Campbell C, Savas P, Nuciforo P, Aura $\mathrm{C}$, et al. Tumor-infiltrating lymphocytes and associations with pathological complete response and event-free survival in HER2-positive early-stage breast cancer treated with lapatinib and trastuzumab: a secondary analysis of the NeoALTTO Trial. JAMA Oncol. 2015;1:448-54.

9. Ingold Heppner B, Untch M, Denkert C, Pfitzner BM, Lederer B, Schmitt W, et al. Tumor-infiltrating lymphocytes: a predic- 
tive and prognostic biomarker in neoadjuvant-treated HER2positive breast cancer. Clin Cancer Res. 2016;22:5747-54.

10. Salgado R, Denkert C, Demaria S, Sirtaine N, Klauschen F, Pruneri $\mathrm{G}$, et al. The evaluation of tumor-infiltrating lymphocytes (TILs) in breast cancer: recommendations by an International TILs Working Group 2014. Ann Oncol. 2015;26:259-71.

11. Denkert C, Wienert S, Poterie A, Loibl S, Budczies J, Badve S, et al. Standardized evaluation of tumor-infiltrating lymphocytes in breast cancer: results of the ring studies of the international immuno-oncology biomarker working group. Mod Pathol. 2016;29:1155-64.

12. Dowsett M, Smith IE, Ebbs SR, Dixon JM, Skene A, A'Hern R, et al. Prognostic value of Ki67 expression after short-term presurgical endocrine therapy for primary breast cancer. J Natl Cancer Inst. 2007;99:167-70.

13. von Minckwitz G, Schmitt WD, Loibl S, Muller BM, Blohmer JU, Sinn BV, et al. Ki67 measured after neoadjuvant chemotherapy for primary breast cancer. Clin Cancer Res. 2013;19: 4521-31.

14. Dieci MV, Criscitiello C, Goubar A, Viale G, Conte P, Guarneri $\mathrm{V}$, et al. Prognostic value of tumor-infiltrating lymphocytes on residual disease after primary chemotherapy for triple-negative breast cancer: a retrospective multicenter study. Ann Oncol. 2014;25:611-8.

15. Hamy AS, Pierga JY, Sabaila A, Laas E, Bonsang-Kitzis H, Laurent $C$, et al. Stromal lymphocyte infiltration after neoadjuvant chemotherapy is associated with aggressive residual disease and lower disease-free survival in HER2-positive breast cancer. Ann Oncol. 2017;28:2233-40.

16. Garcia-Martinez E, Gil GL, Benito AC, Gonzalez-Billalabeitia E, Conesa MA, Garcia Garcia T, et al. Tumor-infiltrating immune cell profiles and their change after neoadjuvant chemotherapy predict response and prognosis of breast cancer. Breast Cancer Res. 2014;16:488.

17. Watanabe $\mathrm{T}$, Hida AI, Inoue N, Imamura M, Fujimoto $\mathrm{Y}$, Akazawa $\mathrm{K}$, et al. Abundant tumor infiltrating lymphocytes after primary systemic chemotherapy predicts poor prognosis in estrogen receptor-positive/HER2-negative breast cancers. Breast Cancer Res Treat. 2018;168:135-45.

18. Miyashita M, Sasano H, Tamaki K, Hirakawa H, Takahashi Y, Nakagawa S, et al. Prognostic significance of tumor-infiltrating CD8+ and FOXP3+ lymphocytes in residual tumors and alterations in these parameters after neoadjuvant chemotherapy in triple-negative breast cancer: a retrospective multicenter study. Breast Cancer Res. 2015;17:124.

19. Arnedos M, Nerurkar A, Osin P, A'Hern R, Smith IE, Dowsett $\mathrm{M}$. Discordance between core needle biopsy $(\mathrm{CNB})$ and excisional biopsy (EB) for estrogen receptor (ER), progesterone receptor (PgR) and HER2 status in early breast cancer (EBC). Ann Oncol. 2009;20:1948-52.

20. Chen X, Zhu S, Fei X, Garfield DH, Wu J, Huang O, et al. Surgery time interval and molecular subtype may influence Ki67 change after core needle biopsy in breast cancer patients. BMC Cancer. 2015;15:822.

21. Mann GB, Fahey VD, Feleppa F, Buchanan MR. Reliance on hormone receptor assays of surgical specimens may compromise outcome in patients with breast cancer. J Clin Oncol. 2005;23:5148-54.

22. Karn T, Metzler D, Ruckhaberle E, Hanker L, Gatje R, Solbach C, et al. Data-driven derivation of cutoffs from a pool of 3,030 Affymetrix arrays to stratify distinct clinical types of breast cancer. Breast Cancer Res Treat. 2010;120:567-79.

23. Tagliabue E, Agresti R, Carcangiu ML, Ghirelli C, Morelli D, Campiglio $\mathrm{M}$, et al. Role of HER2 in wound-induced breast carcinoma proliferation. Lancet. 2003;362:527-33.

24. Goldhirsch A, Winer EP, Coates AS, Gelber RD, Piccart-Gebhart M, Thurlimann B, et al. Personalizing the treatment of women with early breast cancer: highlights of the St Gallen International Expert Consensus on the Primary Therapy of Early Breast Cancer 2013. Ann Oncol. 2013;24:2206-23.

25. Jeselsohn RM, Werner L, Regan MM, Fatima A, Gilmore L, Collins LC, et al. Digital quantification of gene expression in sequential breast cancer biopsies reveals activation of an immune response. PLoS One. 2013;8:e64225.

26. Mathenge EG, Dean CA, Clements D, Vaghar-Kashani A, Photopoulos S, Coyle KM, et al. Core needle biopsy of breast cancer tumors increases distant metastases in a mouse model. Neoplasia. 2014;16:950-60.

27. Vaupel P, Mayer A. Tumor oxygenation status: facts and fallacies. Adv Exp Med Biol. 2017;977:91-9.

28. Globerson A, Effros RB. Ageing of lymphocytes and lymphocytes in the aged. Immunol Today. 2000;21:515-21.

29. Mao Y, Qu Q, Chen X, Huang O, Wu J, Shen K. The prognostic value of tumor-infiltrating lymphocytes in breast cancer: a systematic review and meta-analysis. PLoS One. 2016;11:e0152500.

30. Khan AM, Yuan Y. Biopsy variability of lymphocytic infiltration in breast cancer subtypes and the ImmunoSkew score. Sci Rep. 2016;6:36231. 